\title{
Orbital angular momentum acoustic modes demultiplexing by machine learning methods
}

\author{
D A Stankevich ${ }^{1}$ \\ ${ }^{1}$ Volgograd State University, Universitetskiy prt., 100, Volgograd, Russia, 400062 \\ e-mail: stankevich@volsu.ru
}

\begin{abstract}
Orbital angular momentum (OAM) multiplexing is a promising method for MIMO multiplexing strategy. OAM multiplexing has previously been demonstrated for underwater acoustic communication, where data transmission was carried out within a single acoustic beam. Inner-product method is most often used for OAM demultiplexing, but it is sensitive to changes of signal parameters. For example, parameters changes can be associated with wave propagation through heterogeneous medium. I propose and demonstrate an approach using of machine learning methods to increase demultiplexing accuracy to $96 \%$ for non-stationary signals. In article presents experimental and numerical investigation results of proposed method.
\end{abstract}

\section{Introduction}

Allen [1] first showed that Laguerre-Gauss modes have an orbital angular momentum. Laser beams with an OAM are also called optical vortices. Optical vortices are used in quantum communication [2], particle manipulation [3], metrology [4], etc. A modern review of waves with OAM is given in [5].

The factor $\exp (i l \varphi)$ is responsible for OAM, where $\varphi$ is azimuth angle in a plane perpendicular to the beam axis, $l=0, \pm 1, \pm 2, \ldots$ is topological charge. Thus, the energy flux vector describes a helicoidally surface in space, and beams with different $l$ are characterized by a different distribution of the phase wave front.

Information coding using waves with different OAM consists in multiplexing several orthogonal modes in one beam [6]. This encoding method is widely used for free-space optics communication, where a record data transfer density has already been demonstrated [7]. In [8,9], it is proposed to use waves with OAM for hydro-acoustic communication. For underwater applications increase data transmission rate due by expansion of the spectrum is made difficult by the strong attenuation of sound waves in water.

Decoding information from the spatial distribution of the wavefront is its division on orthogonal modes [6]. Usually demultiplexing is carried out by correlation methods $[6,8]$. Their accuracy can be significantly reduced due to a change carrier frequency of the signal, for example, due to the Doppler effect, in case the receiver or transmitter moves. The machine learning methods could be used for synthesis of OAM demultiplexer when the carrier frequency is a priori unknown or varies. In addition, the amplitude and phase of acoustic wave depends on the temperature of the transmitters. This leads to additional phase front distortions. 


\section{Method of OAM wave demultiplexing}

Arrange $R$ receivers evenly around circumference with center on the beam axis. Then discrete samples of the signal from $r$-th receiver when registering a wave with $l$-th OAM can be represented as:

$$
x_{r l}[n]=A_{l} \sin \left(2 \pi f_{0} n+\varphi_{l}+2 \pi \frac{r l}{R}\right) .
$$

Here I have introduced the following notation: $n=0,1,2, \ldots, N-1, N$ is the number of samples in sample frame, $f_{0}$ is the dimensionless frequency normalized to sampling frequency $f_{d}$, $\varphi_{l}$ is the initial phase of $l$-th mode. The amplitudes of the $A_{l}$ signals are normalized so that the signal with several modes has same energy as the signal with a single mode.

Following [8], to encode an information symbol with a size of one byte, we will use the sum of helicoidal waves with $l$ from -4 to 4 :

$$
x_{r}[n]=\sum_{l=-4}^{4} A_{l} \sin \left(2 \pi f_{0} n+2 \pi l \frac{r}{R}+\varphi_{r}\right)+\eta_{r}[n] .
$$

The amplitude $A_{l}$ is assumed to be zero if the corresponding bit is not set (see Figure 1). A wave with zero orbital momentum encodes a zero byte value.

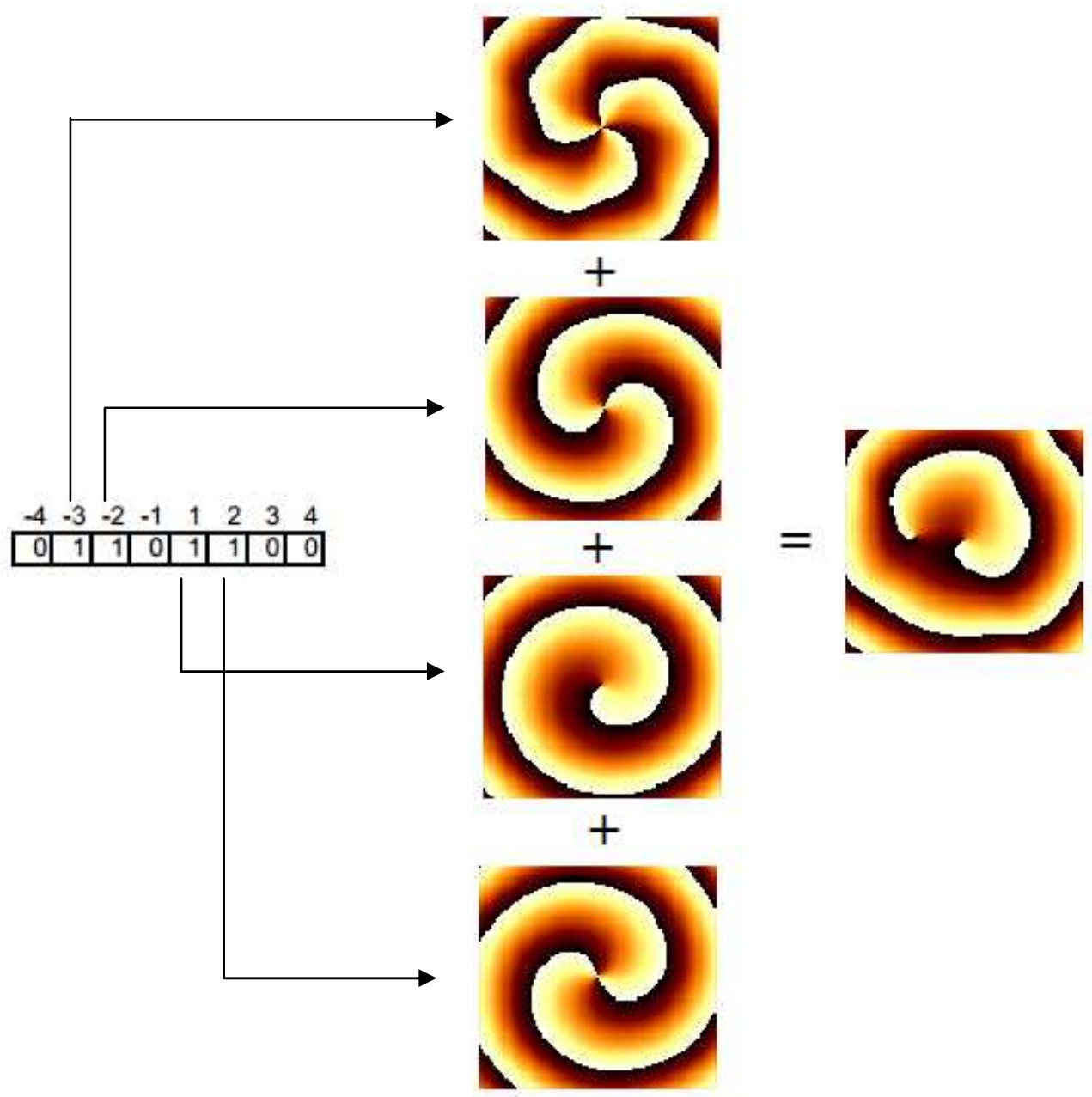

Figure 1. Multiplexing process of orthogonal modes for one byte.

A two-layer convolution network $[10,11]$ was used for OAM demultiplexing. A description of its architecture is given in Table 1 and shown on Figure 2. The first layer contains $M=10$ convolution neurons that have $R$ inputs: 


$$
y_{m}^{(1)}[n]=\operatorname{ReLU}\left(\sum_{r=0}^{R-1} \sum_{k=0}^{K-1} w_{m r}^{(1)}[k] x_{r}[k+n]+b_{m}^{(1)}\right),
$$

Here $w_{m r}^{(1)}, b_{m}^{(1)}$ are weights and biases of first convolution layer. Input layer has nonlinear ReLU (rectified linear unit) activation function: $\operatorname{ReLU}(x)=\max (0, x)$.

Table 1. Neural network architecture.

\begin{tabular}{ccc}
\hline & Input layer & Output layer \\
\hline Layer type & Conv1D & Dense \\
Neurons & 10 & 8 \\
Convolution kernel & $10 \times 1$ & - \\
Pooling & $5 \times 5(\mathrm{Max})$ & - \\
Activation & ReLU & Sigmoid \\
\hline
\end{tabular}

Results thinning of input layer (pooling) is used to reduce the number of connections with output layer and to increase learning speed. The sequence from outputs of neuron filters is divided into disjoint intervals of 5 points, and the maximum value is selected at each interval.

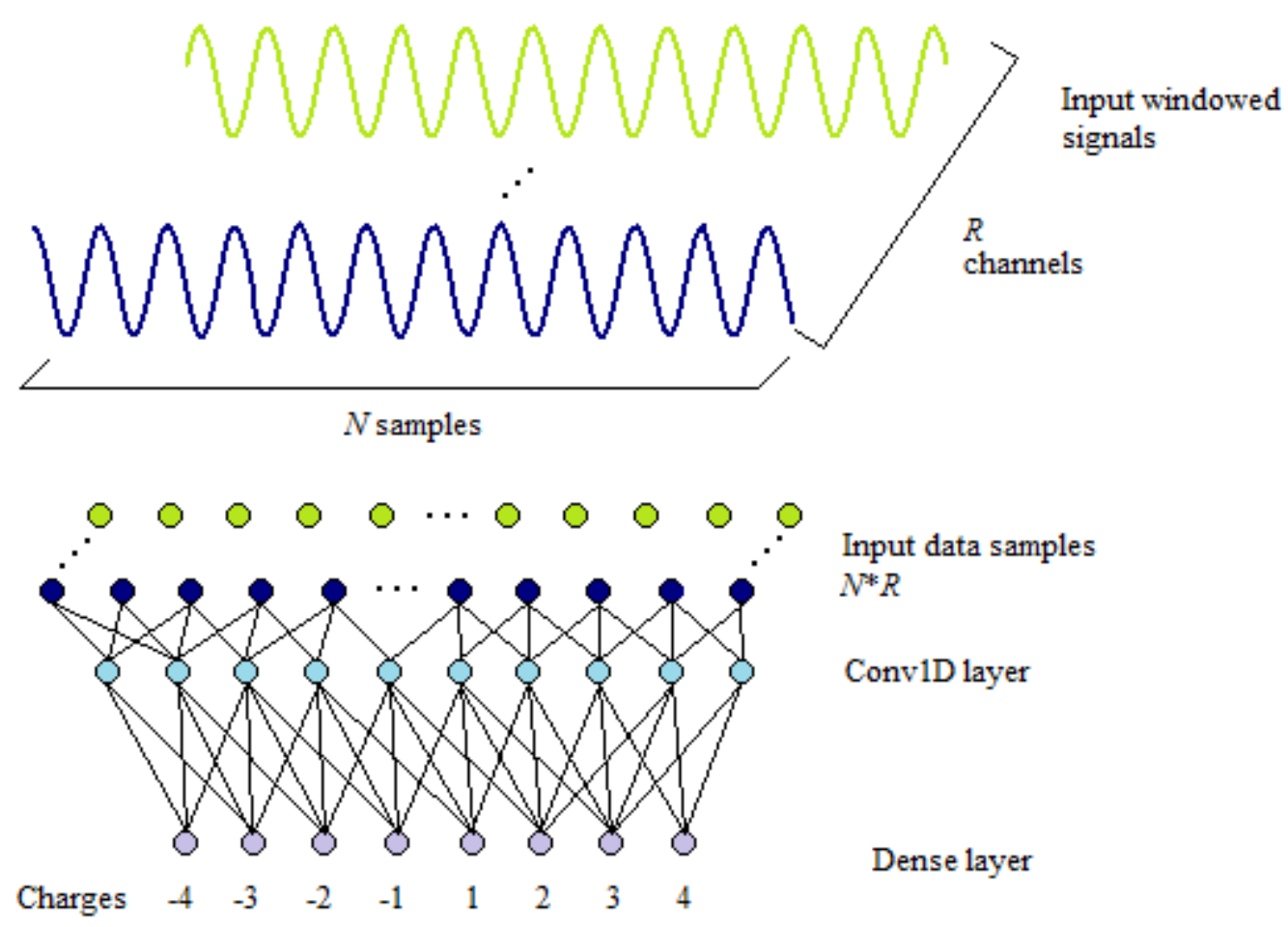

Figure 2. Architecture of the neural network.

Further, these data proceed to the output fully connected layer consisting of 8 neurons $(q=0-7)$ with a sigmoidal activation function: 


$$
\hat{p}_{q}=\left(1+\exp \left(-b_{q}^{(2)}-\sum_{n=0}^{N-1 M-1} \sum_{m=0}^{(2)} w_{q n m}^{(1)} y_{m}[n]\right)\right)^{-1}
$$

Thus, the activation $\left(\hat{p}_{q}>0.5\right)$ of output neuron $q$ indicates the presence of a mode with given angular momentum. Note that several neurons can be activated.

The network weights are tuned in the learning process on a train set consisting of 2000 signals corresponding to different values of information symbols. Every signal consist $N=20$ samples with additive normal noise with zero mean and dispersion $\sigma^{2}$, randomly selected from range $1.0-2.0$. The initial phase and frequency of signals are uniformly distributed in range of $0-2 \pi$ and $0.1-0.3$, respectively.

Training is carried out by back-propagation error method with Keras [12] deep learning library. In the process of learning, consisting of 100 epochs, binary cross entropy was minimized with Adam [13] method. Training time on a 12-core Intel $\mathrm{i} 7-3930 \mathrm{~K} 3.2 \mathrm{GHz}$ processor is less than $30 \mathrm{~s}$.

The study of network characteristics was carried out on a different set, containing also 2000 signals. With a relatively low noise level $\left(\sigma^{2}=0.1\right)$, ten receivers are enough to achieve $100 \%$ accuracy of demultiplexing (see Figure 3a). A similar result is achieved using the correlation method. Increasing noise variance by an order reduces accuracy, but it remains at level of $96 \%$.

Accuracy of demultiplexing by the proposed method in a sufficiently wide frequency range $(0.15-$ 0.3 ) stay not lower than $91 \%$ (see Figure 3b). As opposed the correlation method loses significantly in accuracy when the signal frequency changes by $10 \%$.

a)

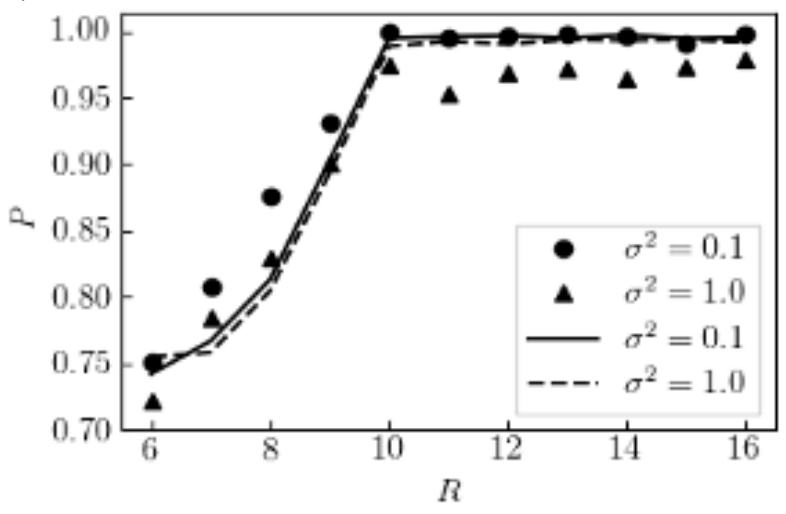

b)

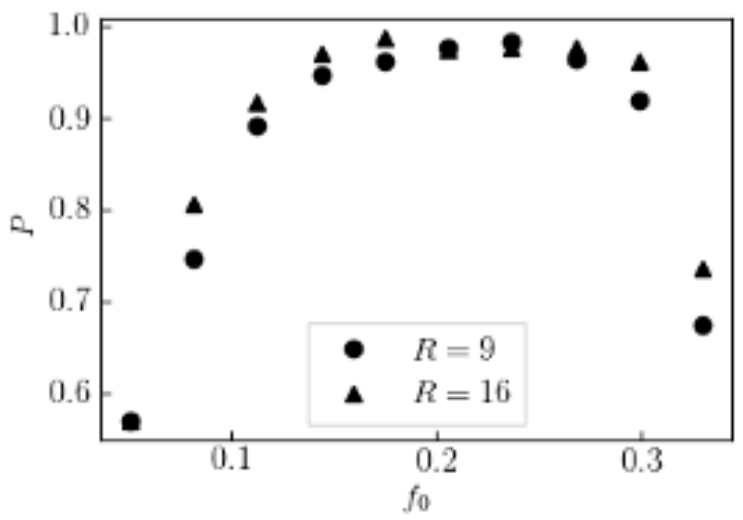

Figure 3. a) Demultiplexing accuracy dependencies $P$ by receivers count $R$ for neural net (circle and triangles) and for correlation method (line); b) demultiplexing accuracy dependencies $P$ by carrier frequency $f_{0}$ of signal $\left(\sigma^{2}=1.0\right)$.

\section{Experimental results}

An experimental setup was created to transmit information using two identical transducers array. Each array consisted of 7 piezoelectric transducers (type ZP-1) (see Figure 3). One array was connected to output of the sound card (UMC1820), and the other to its input. Transducers are placed into boxes from corrugated cardboard with a thickness of $4 \mathrm{~mm}$. Noise insulation "Penolon" with a thickness of $10 \mathrm{~mm}$ is glued inside the boxes, and their internal volume is filled with cotton. The front panels are made of MDF and also covered with sound insulation (Figure 4). Piezo-transducers are mounted on a 
round fiberglass board (1.6 $\mathrm{mm}$ thick and $164 \mathrm{~mm}$ in diameter), in which eight round holes of $36 \mathrm{~mm}$ in diameter are milled. Central transducer was not used in experiments.
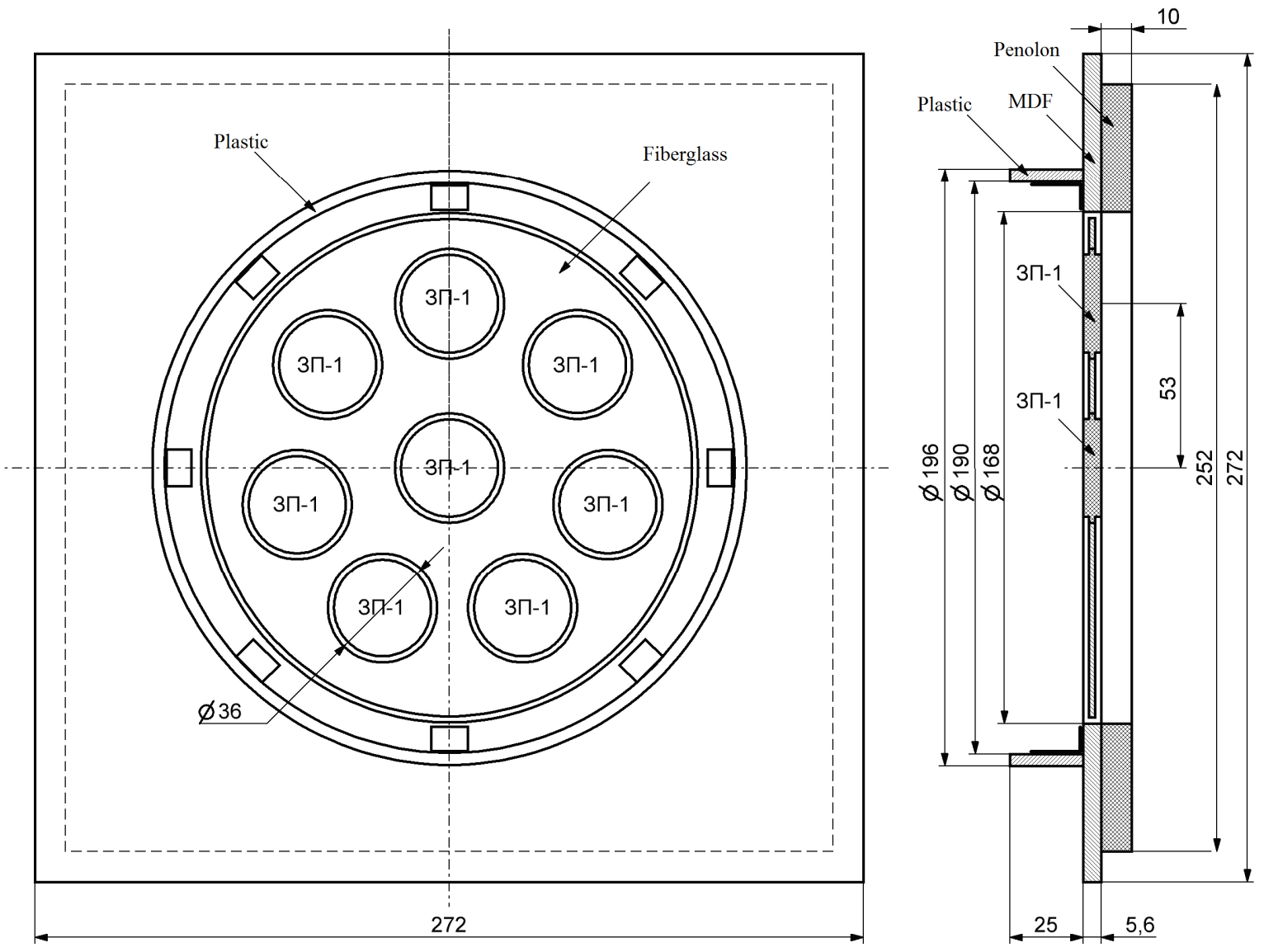

Figure 4. Transducers array front panels.

The Figure 5 shows the experimentally obtained pressure and phase distributions for waves with different topological charges. Since the amplitude-frequency response characteristic of the transducers differ, it is clear that the wave front is slightly different from the ideal helicoidal one. It is especially noticeable for higher order topological charges (see Figure 6). This circumstance required slightly different network architecture (Table 2) in order to achieve high accuracy.

Table 2. Modified neural network architecture for experiments.

\begin{tabular}{ccc}
\hline & Input layer & Output layer \\
\hline Layer type & Conv1D & Dense \\
Neurons & 50 & 6 \\
Convolution kernel & $200 \times 1$ & - \\
Activation & Leaky ReLU & Sigmoid \\
\hline
\end{tabular}



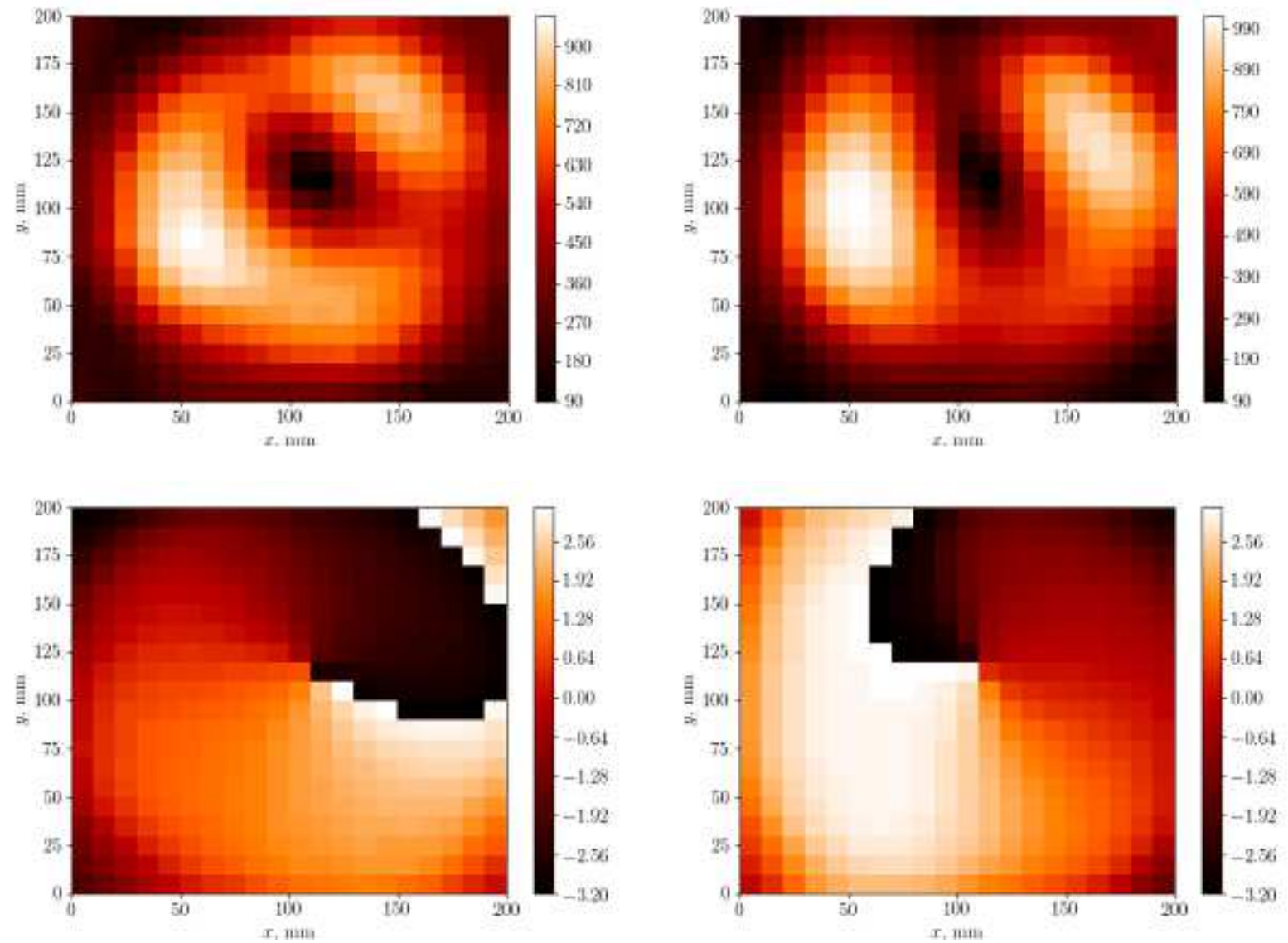

Figure 5. Experimental amplitude (top) and phase (bottom) of pressure distributions for waves with a topological charge +1 (left) and -1 (right).
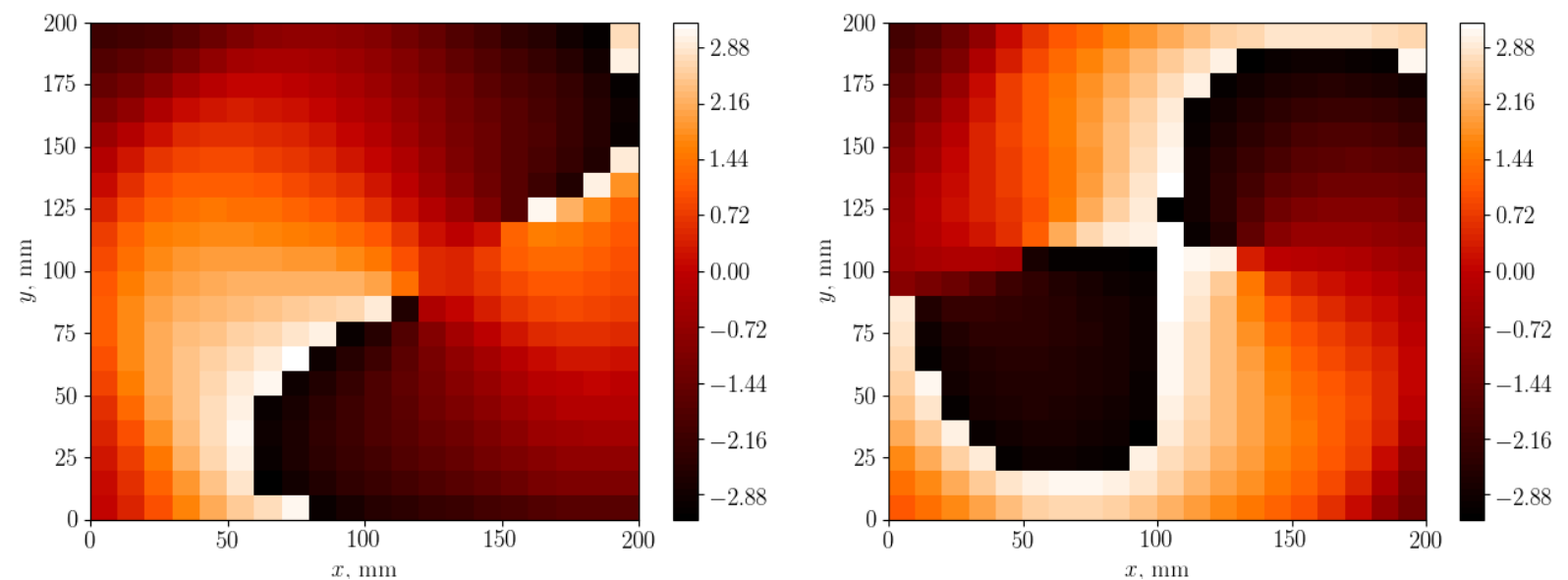

Figure 6. Experimental phase of pressure distributions for waves with a topological charge +2 (left) and -2 (right). 
Real signals obtained in the experiment used for training. Each signals consisted of 400 samples. The training cycle consisted of two stages. At first, 20 signals with a random set of bits (training set) were transmitted. Then 10 signals were transmitted, according to which the accuracy of information transfer was assessed (test set).

The receiver and transmitter were located at a height of $0.5 \mathrm{~m}$ from the floor. Signal information $(6$ bits) transmits at a frequency of $2.3 \mathrm{kHz}$ by topological charge coding with $l=-3 \ldots 3$. The training consists of 100 cycles. At a distance of $2 \lambda$, accuracy of demultiplexing (using the developed neural network demultiplexer) was $100 \%$, and at a distance of $17 \lambda$, it was $96 \%$. Transmission accuracy is reduced primarily due to the presence of significant reflections from the walls and floor.

\section{Conclusion}

The paper presents results of numerical and experimental studies of neural network demultiplexing method for wave with orbital angular momentum. It is shown that proposed method gives a significant advantage over the classical correlation method. Usually systems using neural networks are not high performance. However, the small number of layers of the developed architecture makes it possible to solve the problem of demultiplexing on a simple signal processor.

\section{References}

[1] Allen L, Beijersbergen M W, Spreeuw R J C.and Woerdman J P 1992 Orbital angular momentum of light and the transformation of Laguerre-Gaussian laser modes Phys. Rev. A 45 8185-8189 DOI: 10.1103/PhysRevA.45.8185

[2] Erhard M, Fickler R, Krenn M and Zeilinger A 2018 Twisted photons: new quantum perspectives in high dimensions (Light: Science \& Applications) 717146 DOI: 10.1038/ lsa.2017.146

[3] Hong Z Y, Yin J F, Zhai W, Yan N, Wang W L, Zhang J and Drinkwater B W 2017 Dynamics of levitated objects in acoustic vortex fields Scientific Reports 7093 DOI: 10.1038/s41598-017-07477-1

[4] Yue Z, Ren H, Wei S, Lin J and Gu M 2018 Angular-momentum nanometrology in an ultrathin plasmonic topological insulator film Nature Communications 94413 DOI: 10.1038/s41467-018-06952-1

[5] Chen R, Zhou H, Moretti M, Wang X and Li J 2019 Orbital Angular Momentum Waves: Generation, Detection and Emerging Applications arXiv: 1903.07818

[6] Djordjevic I B 2017 Multidimensional OAM-Based Secure High-Speed Wireless Communications IEEE Access 17140317 16416-16428 DOI: 10.1109/ ACCESS.2017.2735994

[7] Li L, Zhang R, Zhao Z, Xie G, Liao P, Pang K, Song H, Liu C, Ren Y, Labroille G, Jian P, Starodubov D, Lynn B, Bock R, Tur M and Willner A E 2017 High-Capacity Free-Space Optical Communications Between a Ground Transmitter and a Ground Receiver via a UAV Using Multiplexing of Multiple Orbital-Angular-Momentum Beams Scientific Reports 717427 DOI: 10.1038/s41598-017-17580-y

[8] Shi C, Dubois M, Wang Y and Zhang X 2017 High-speed acoustic communication by multiplexing orbital angular momentum PNAS 114(28) 7250-7253 DOI: 10.1073/ pnas. 1704450114.

[9] Hefner B T, Marston P L 1999 An acoustical helicoidal wave transducer with applications for the alignment of ultrasonic and underwater systems J. Acoust. Soc. Am. 106(6) 3313-3316 DOI: $10.1121 / 1.428184$ 
[10] Goodfellow I, Bengio Y and Courville A 2016 Deep learning (MIT Press) p 775

[11] Bolotova Y A, Spitsyn V G and Osina P M 2017 A review of algorithms for text detection in images and videos Computer Optics 41(3) 441-452 DOI: 10.18287/ 2412-6179-2017-41-3-441-452

[12] Dua R, Ghotra M S 2018 Keras Deep Learning Cookbook: Over 30 recipes for implementing deep neural networks in Python (Packt Publishing) p 252

[13] Kingma D, Ba J 2014 Adam: A Method for Stochastic Optimization arXiv: 1412.6980

\section{Acknowledgment}

This investigation was supported by the Russian Science Foundation, project no. 18-79-00080. 